\title{
Elemente einer systemtheoretischen Betrachtung des Stoffwechsels ${ }^{1}$
}

\author{
Alfred LOCKER \\ Institut für Strablenscbutz, Reaktorenzentrum Seibersdorf-Wien, Österreich \\ (Österreichische Studiengesellschaft für Atomenergie GmbH)
}

\begin{abstract}
Elements of a systems-theoretical approach to metabolism. The discussion of general formal properties of metabolizing systems reveals the justification of applying tentatively General Systems Theory as a most general background. Whereas set-theoretical and graph-theoretical considerations, because of their very abstract nature, are obviously not well suited, the application of the theory of compartmented systems, elaborated on the basis of network or linear systems theories, seems to be useful. In metabolic studies physical models in the sense of computers operating as simulators are indispensable tools for research. A comparison between cybernetic and kinetic models demonstrates the many links and similarities rather than contrasts existing between them. Some epistemological problems are also included in the extended discussion of the concepts of information and energy, basical for these models.
\end{abstract}

\section{ÜBERBLICK ÜBER DEN GANG DER BETRACHTUNG}

Den Stoffwechsel als ein organisiertes, dynamisches, regulatives und autoregulatives Geschehen vom Standpunkte einer theoretischen Konzeption zu sehen, die in allgemeinster Form Aussagen über komplexe Phänomene und ihre Grundlagen zu machen imstande ist, wird nach der heute fast völligen Ausschöpfung der primären (elementaren) biochemischen und physiologischen Gegebenheiten nahegelegt. Die Anwendung einer Theorie auf komplexe reale Vorgänge erhellt zwar letztere außerordentlich, führt aber schließlich auch zur Verfeinerung der theoretischen Vorstellungen selbst, gleichsam als ein im Erkenntnisprozeß verankerter Rückkoppelungsmechanismus. Die Heranziehung der Allgemeinen Systemtheorie stellt in dieser Hinsicht kein Novum dar, als alle bisherigen Ansätze zur theoretischen Interpretation von Stoffwechselvorgängen auf dem Boden von Konzeptionen stehen, die ihrerseits in bestimmter Weise mit der Systemtheorie als deren speziellere Ausgestaltungen in Verbindung stehen.

So wird unsere Betrachtung sich nicht nur ganz allgemein mit den Begriffen (und der aus ihnen entwickelten Theorie) von "System" und „Modell“ sowie einigen hierher

1 Hern Prof. Dr. L. v. BertalanfFy, meinem hochverehrten Lehrer, zu seinem 65. Geburtstag gewidmet. 
gehörigen epistemologischen Folgerungen auseinandersetzen; sie wird auch durch die Erörterung von teilweise komplementären Begriffen bestimmt, die wie „informational “ und "strukturell", "deterministisch" und "stochastisch" zu einer Spezifikation der Behandlung der fundamentalen Begriffe "System " und "Modell" führen und damit aud deren Ubertragung auf allgemeine Probleme des Stoffwechsels beeinflussen. Der Vergleich, die Gegenüberstellung und Verbindung von „kybernetischen “ und „kinetischen“ Modellen des Stoffwechsels ermöglicht uns unter anderem den Zugang zu konkreteren Problemen, für den auch die Diskussion der Anwendung von Computern Bedeutung haben mag. Unsere notwendigerweise gedrängte Darstellung ist von dem Bestreben geleitet, der quantitativen Biologie des Stoffwechsels, deren Grundprobleme schon früher dargelegt wurden (Locker 1964), die für ihren Ubergang aus dem empirischen Bereich - in dem sich bloß phänomenologische Gesetze abzeichnen - in den Bereich sicherer theoretischer Fundierung erforderlichen Grundlagen wenigstens andeutungsweise $\mathrm{zu}$ benennen.

\section{SYSTEM IN ABSTRAKTER UND KONKRETER BETRACHTUNG}

\section{Einige Ergebnisse der Allgemeinen Systemtheorie}

Vieles in der uns umgebenden Wirklichkeit imponiert als System. Rein abstrakt und allgemein ist ein solches mit v. BerTALANFFY (1950a) als ein Komplex von in Wechselwirkung stehenden Elementen aufzufassen, als ein in sich gegliedertes Ganzes also, wie denn auch der Systembegriff überhaupt der wissenschaftich legitime Erbe des umstrittenen Ganzheitsbegriffes ist (LockER 1966). Unter Rückgriff auf gewisse mengentheoretische Vorstellungen geben HALL \& FAGEN (1956) eine allgemeine Definition von „System“: „Ein System ist eine Menge von Objekten zusammen mit den Beziehungen zwischen diesen und zwischen ihren Eigenschaften", die trotz der Möglichkeit, typische Eigentümlichkeiten von Systemen, die bei isolierter ebenso wie bei vergleichender Betrachtung zutage treten, wie unter anderem Zentralisation, Adaptation, Isomorphien, Zustandsbestimmtheit etc., verständlich zu machen, direkte Prinzipien der Anwendbarkeit auf konkrete Gegebenheiten - das Anliegen unserer Untersuchung - nicht erörtert. Auch die strenge mengentheoretische Definition MESARovics (1964) bleibt im wesentlichen insofern abstrakt, als sie keine direkte Verbindung zur uns interessierenden Anwendung liefert, aber doch zu allgemeinen Schlußfolgerungen kommt, die indirekt auch für biologische Probleme und den Stoffwechsel Bedeutung haben. Die zuerst von v. BERTALANFFY (1950b) herausgestellte Unterscheidung $z$ wischen „geschlossenem" und "offenem" System, fundamental für die Biologie, läßt sich auf mengentheoretischer Basis streng durchführen, der Begriff der Struktur als das herausarbeiten, was nach Wegfallen der Bestandteile an Relation übrigbleibt und als die für die Systemfunktion wichtigsten Begriffe lassen sich die von „input" (Eingang), "output“ (Ausgang) und „Zustand“ gewinnen.

Obwohl im mengentheoretischen Ansatz auch Zeitfunktionen betrachtet werden, scheint sich eine brauchbarere Beziehung zwischen Allgemeiner Systemtheorie und Stoffwechsel zu ergeben, wenn erstere im Gewand der "relationalen Biologie" 
(RASHEVsky 1954, Rosen 1958a, b), einer im wesentlichen topologischen und graphtheoretischen Darstellung, auftritt. Hier ergibt sich nämlich bereits eine ganz allgemeine Definition des Stoffwechsels als eine Folge von Operationen, durch welche ein aus der Umgebung stammender Satz von Material (,input“-Material) in einen neuen Satz von Material ("output"-Material) umgewandelt wird. Die Struktur, durch welche die "input-output"-Umwandlung bewirkt wird, ist das System. Von der Grob-Struktur, die, solange sie selbst innerlich als nicht weiter auflösbar betrachtet werden muß, "black-box"-Charakter zeigt, kann zur Feinstruktur fortgeschritten werden, also zu den Komponenten, die untereinander durch „input-output“-Relationen verbunden sind. Es liegt im Gange der Forschung, daß die „Korngröße“ der Feinstruktur immer weiter verringert wird, obgleich es sicher ist, daßs auch die jeweils "letzten" Einheiten, die gewonnen werden können, „black-box"-Natur beibehalten. In der Auftrennung von Grobstruktur und Feinstruktur findet sich der von RASHEvsky (1960) geprägte Unterschied zwischen "relational " und "metrisch" wieder.

Beim so betrachteten System kann die Abgrenzung vieler bedeutsamer Eigenschaften, wie von Original-Komponenten, die den „input“ aus der Umgebung aufnehmen, und von Terminal-Komponenten, die den „output" an die Umgebung abgeben, sowie die Möglichkeit der Auflösung in Subsysteme, die keinen „input“ von einer nicht im System enthaltenen Komponente bekommen, nicht über die Nachteile hinwegtäuschen, die sich bei der graph-theoretischen Darstellung im Blockdiagramm einstellen. So wird über die Frage, welche Komponenten des Systems für die „Operation" wichtig sind und welche nicht, keine ausreichende Festsetzung gemacht; die zeitlichen Verzögerungen, die wie der Operations-",lag“ als Eigenschaft der Komponenten und der Transport-„lag" bei der Ubertragung zwischen den Komponenten auftreten, erfahren zunächst keine ausreichende Behandlung. Systeme dieser Art werden $M R$-Systeme genannt, da sie wegen der endlichen Lebensdauer der Komponenten $(M)$ deren Ersatz durch Reparation $(R)$ erforderlich machen. Ein weiterer zunächst in Erscheinung tretender Nachteil des Diagramms ist das Fehlen der Eindeutigkeit; wenn zwei Pfeile (gerichtete Kanten) aus einer Komponente (etwa $M_{l}$ ) ausgehen, läßt sich nicht sagen, ob sie differente "outputs" repräsentieren oder ob der gleiche "output ${ }^{\text {" }}$ zwei verschiedenen Komponenten zufließt. Diese Nachteile können unter Verwendung des begrifflichen Rüstzeugs der Kategorien-Theorie (EILEnberg \& MACLANE 1945) durch sogenannte „kanonische Diagramme" und „kanonische Repräsentation“, vor allem aber durch Uberführung der Darstellung in die der Netzwerktheorie überwunden werden. So wird trotz Weiterführung der abstrakten Relationalen Biologie (RAsHevsKy 1965) durch die abstrakten Blockdiagramme der Netzwerktheorie ein Ubergang zur direkten kinetischen Behandlung des Stoffwechsels durch letztere möglich (Schoenfeld 1963). Besonders im Hinblidk auf konkrete Probleme des Stoffwechsels sind bestimmte weitere mathematische Verfahren angewandt worden, auf die wir noch kurz zu sprechen kommen werden. 


\section{Die epistemologische Bedeutung der Einbeziehung des Beobachters in das System}

Nicht nur "nach unten", von Grobstruktur zu Feinstruktur, besteht Auflösbarkeit eines Systems, es ist auch eine Erweiterung "nach oben" durchführbar, zu einer Art

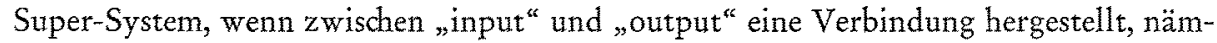
lich eine Feedback-Schlinge eingefügt wird, in welcher oder mittels derer ein Beobachter agiert (Abb. 1). Die Beobachtung besteht im Vergleich des Resultats eines „input“Satzes mit einem anderen; zunächst befindet sich der "input" in Zustand $l$ und der "output" im Zustand S (AsHBy 1961). Durch Beobachtung und Experiment (Veränderung der „input“-Menge) tritt eine Veränderung des „output“ in $S^{\prime}$ ein. Infolge der

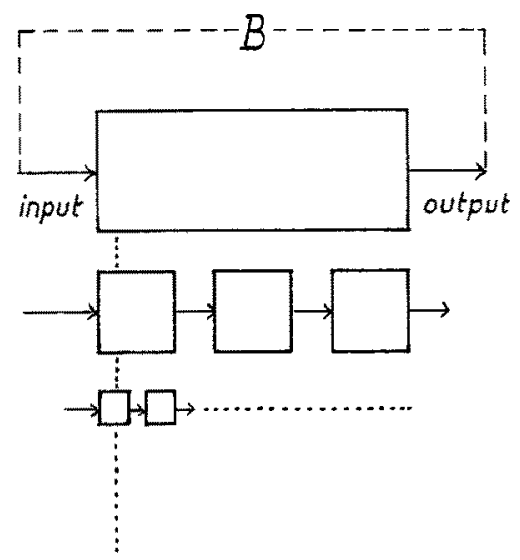

Abb. 1: System (Modell) als „black box“. Auflösung in Komponenten, gleichfalls mit „black box"-Charakter, durch den Fortgang der Forschung. Ubergang zu einem Super-System durch Einbeziehung des Beobachters (B) in die Feedback-Verbindung von „output" $z u$,input"

„black-box“-Natur des Systems ist nur die Transformation (die Abbildung, „mapping") der „input"-Zustände in „output"-Zustände registrierbar, wenn auch die Forderung erhoben werden kann, daß dieses "mapping“ ideal die gesamte Information über die „black-box" enthalten muß, so daß es alle Beobachtungen über das System repräsentiert. Aus der Interaktion des Beobachters mit dem (ursprünglichem) System, weil er sich während der Beobachtung sozusagen außerhalb oder innerhalb dieses befinden kann, bzw. auch je nachdem, ob der Schwerpunkt der Betrachtung auf das (ursprüngliche) System oder den Beobachter gelegt wird, kann ein "terminales“ oder "zielsuchendes" Verhalten des Systems (Mesarovic 1964) resultieren. Ersteres kommt dadurch zustande, daß das „mapping “ einfach die gesamte Repräsentation des Systems umfaßt, letzteres auf die Weise, daß das "mapping" gezielt betrieben wird, das heißt

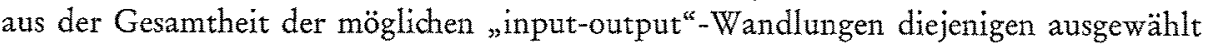
werden, die zu einem ganz bestimmten gewünschten „output" führen. Das erstgenannte Verfahren oder Verhalten kann demnach in etwa mit dem Begriff der Steuerung, das letztgenannte mit dem der Regelung in Beziehung gebracht werden. 


\section{Kontinuierliche und binär-logische "input-output"- W a d lung}

Werden die mit der Umwelt (oder dem Beobachter) in Beziehung stehenden Terminal-Komponenten als Rezeptor bzw. Effektor angesehen, so kann speziell von einem informationsverarbeitenden System gesprochen werden, bei welchem auch das Feedback von letzterem zu ersterem die "Zielsuche" ermöglicht. Die Einführung des Begriffes der Information in die Darstellung rechtfertigt sich hier nur insoweit, als informationstheoretisch (kybernetisch) die „input-output"-Wandlung besonders gut bearbeitet ist, obwohl für die kinetische Betrachtung, bei Bedachtnahme auf die stofflichen Gegebenheiten dieser Transformation, die gleiche formale Beziehung zugrunde gelegt werden muß. Arbeitet das System logisch, kann der Rezeptor als Analog-DigitalWandler angesprochen werden, sofern aus der Umwelt analog variierende Signale

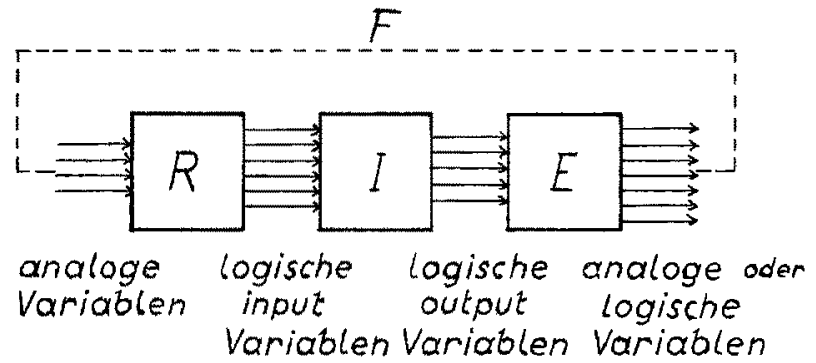

Abb. 2: Modell eines informationsverarbeitenden Systems (nach ZEMANEK et al. 1961, umgezeichnet und vereinfacht). R: Rezeptor, I: Informationsverarbeitung, E: Effektor. Angenommene Wandlung der analogen Umweltvariablen in logische (vice versa). In I können $n$ logische "input"-Variablen (die $2^{n}$ input-Situationen repräsentieren) in $m$ logische "output"Variable ( $2^{m}$ output-Situationen) transformiert werden. Äßere Feedbackschlinge (F) von E nach $R$.

(„inputs") eintreffen; auch die Transformation durch die zwischen Rezeptor und Effektor liegenden Systemkomponenten kann von gleicher Art, aber auch beispielsweise rein logisch sein (Abb. 2). Hier sei darauf hingewiesen, daß im Begriffspaar "analogdigital" keine fundamentale Antithese erblickt werden darf, handelt es sich doch bei einer Wandlung nach dieser Art nur um einen von verschiedenen transformierenden Vorgängen, neben dem kontinuierlich-analoge und diskontinuierlich-analoge Transformation abgegrenzt werden müssen. Bei ersterer wird eine kontinuierlich variierende Quantität durch eine andere kontinuierlich variierende repräsentiert - wie schließlich Zählverfahren und positionale Verfahren, bei welchem die Quantität des Signals nicht durch die Zahl der Impulse, sondern durch ihre Lage in Raum und Zeit gegeben ist (Zemanex, KRetz \& ANgYan 1961). Selbst bei logischer Transformation ist zwischen kombinatorischer (ohne Zeit) und sequentialer Logik (mit quantifizierter Zeit) zu unterscheiden.

Auf Stoffwechselprobleme binäre Logik angewandt zu haben, ist das Verdienst SUGITAs (1961); kinetisch zu beschreibende Vorgänge wurden so schaltalgebraisch dargestellt, unbeschadet der Tatsache, daß sie auch durch hydrodynamische und elektro- 
nische Analoge veranschaulicht werden können. Zwei Ubberlegungen erscheinen hier angebracht: Auch für logische „informationale" Bedingung von Vorgängen kann im Stoffwechsel nur ein materieller Träger (Zeichen-Träger, Signal) vorhanden sein, wenn auch zwischen diesem und dem ausgelösten Vorgang keine stöchiometrische Relation besteht. Weiterhin kann gerade an Hand eines logischen (hydrodynamischen) AnalogModells, wie es SugITA entworfen hat, der Übergang von „analog“ zu „digital" dargestellt werden. Gemäß der Thermodynamik irreversibler Prozesse wird mit dem Flïssigkeitsbehälter (als Veranschaulichung des Intensitätsfaktors) eine Klappe (als Entsprechung zum Geschwindigkeitskoeffizienten in der Beziehung $J=L \cdot X$ zwischen Flüssen und Kräften) in Verbindung gebracht. Es ist nun selbstverständlich, daß außer einer Klappe, die für „on-off“-Kontrolle konstruiert ist, auch eine Konstruktion für ein Kontinuum von Zwischenstellungen zwischen „on “ und „off“ gegeben werden kann und daß insbesondere zwischen „analog" und „digital" eine Wechselwirkung eintritt, wenn $\mathrm{z}$ wei entsprechende Klappen vorhanden sind.

\section{Konkrete Systeme (Kompartimentalsysteme) und ihre mathematische Behandlung}

Von der Darstellung allgemeiner und abstrakter Systeme kann, wie gezeigt, auf mathematische Verfahren zurückgefunden werden, die sich zur Behandlung mehr konkreter Systeme, solcher nämlich, die bei Stoffwechselfragen auftreten, eignen. Zur mathematischen Kennzeichnung des Systemverhaltens eignen sich Differentialgleichungen - im Hinblick auf deren Eigenschaften auch zwischen linearen und nicht-linearen Systemen unterschieden wird - ferner Frequenzgang, Ubergangsfunktion und Übertragungsfunktion (Transfer-Funktion). Die Ubertragungsfunktion kann als die Abbildung der Menge der Paare aus je einem Eingangssignal und einem inneren Zustand in die Menge der inneren Zustände, die Ergebnisfunktion durch die Abbildung derselben Verbundmenge in die Menge der Ausgangssignale charakterisiert werden (KäMMERER 1966). Ein hier unerläßliches mathematisches Hilfsmittel stellt die LAPLACE-Transformation dar. Die mit den am besten ausgearbeiteten mathematischen Verfahren behandelten konkreten Stoffwechselsysteme sind jene, die vor allem bei der Tracer-Kinetik im Gesamtorganismus, aber auch auf zellulärem Niveau in Betracht gezogen werden müssen, die Kompartimentalsysteme.

Im allgemeinen lassen sich bei deren Analyse zwei Grundtypen auseinanderhalten: das "Mamillarsystem“, bei dem ein zentrales Kompartiment mit einer Anzahl von peripheren kommuniziert, und das "Katenarsystem", das mit seiner reihenweisen Anordnung von Kompartimenten besonders als Stoffwechselsystem in Frage kommt (SHEPPARd \& HouseHolder 1951). Unter Kompartiment wird eine in sich homogene Komponente eines Systems verstanden, deren Homogenität sich auf eine bestimmte Variable bezieht, das heißt mittels derer ein Punkt des Kompartiments nicht von einem anderen unterschieden werden kann, während die Unterscheidung verschiedener Kompartimente voneinander durch ihre chemischen Zustände oder ihre Lage möglich ist (RESCIGNO \& SEGRE 1962). Auf Grund einer verallgemeinerten, der ,input-output“Relation entsprechenden "precursor-product"-Beziehung wurde eine weitgehende Klas- 
sifizierung von Kompartimentalsystemen erreicht (REscigno \& SEGRE 1961); unter Annahme von vorwiegender Kinetik 1. Ordnung wurde die mathematische Behandlung auf der Basis der Netzwerktheorie (Rescigno \& SEGRE 1962), wonach ein Knoten der LAPLACE-Transformation der Konzentration oder spezifischen Aktivität, ein gerichteter Arm der Transfer-Funktion zwischen zwei Knoten entspricht, bzw. der Theorie linearer Systeme (HEaron 1963) durchgeführt.

\section{DIE BEDEUTUNG VON MODELLEN}

\section{Abstrakte und konkrete Modelle}

Nur im Hinblick auf die intime Verbindung zur Anwendung kann ein Kompartimentalsystem als tatsächliches, sogar konkretes "System" angesehen werden. Schon die hier methodisch erforderliche Herausstellung der „black-box"-Natur des Kompartiments zeigt, daß es sich bei ihm eigentlich um ein „Modell“ handelt, stellt es doch trotz aller Konkretheit eine Repräsentation der Wirklichkeit dar, die nur auf simplifizierendem (und abstrahierendem) Wege gewonnen werden konnte. Dies gilt immer von der Stellung des Modells zum System, daß ersteres von letzterem wegen dessen Kompliziertheit und Komplexität nur einen Teilaspekt vorstellen kann. Voraussetzung ist die zwischen beiden existierende Analogie, das heißt Aquivalenz in den entscheidenden (strukturellen und funktionellen) Bezügen. Daher ist das Modell auf jeden Fall einfacher als das System, mag es sich nun um ein abstraktes mathematisches Modell - dessen allgemeine Formulierung und Prüfung von BERMaN (1963) diskutiert worden ist oder um ein physikalisches Modell handeln. Können wir zwischen beiden, dem mathematischen und dem physikalischen Modell, immerhin Abstufungen von Abstraktem und Konkretem feststellen - weshalb wir ein Kompartimentalsystem trotz seiner mathematischen Natur wegen seiner perfektionierten Anwendbarkeit als konkret bezeichneten, was vielleicht nicht ganz gerechtfertigt ist - so ist sicherlich das konkreteste physikalische und zugleich flexibelste Modell der Computer, der in seiner Bedeutung mit gewisser Ausführlichkeit gewürdigt werden soll.

\section{Computer-Modelle}

Von den beiden Seiten der Computer-Verwendung als eines Rechengeräts oder eines Simulators interessiert hier die zweite, während die von Chance, Higgrns \& Garfinkel (1962) erörterte Abgrenzung der Vor- und Nachteile von Analog- und Digitalcomputern für den prinzipiellen Modellcharakter des Computers ohne Belang ist. Wenn Gleichungssysteme einmal durch den Computer gelöst sind, kann durch Änderung der Variablen-Werte mit ihm experimentell gearbeitet werden, ebenso wie durch geringe Anderung des Gleichungssystems selbst schnell der Effekt eines neuen Satzes von Annahmen geprüft werden kann. Ist einmal das einem kinetischen Schema entsprechende Flußdiagramm entworfen und, wie beim Digitalcomputer, automatisch in seine eigene „problemorientierte Sprache“, zum Beispiel FORTRAN übersetzt, dann simu- 
liert dieser durch die Aufstellung und Lösung der Differentialgleichungen das in der Annahme gegebene System. Er kann sonach Multi-Kompartimentalsysteme darstellen, wie sie sich in der Tracer-Kinetik oder Pharmako-Kinetik (TAYLoR \& WIEgand 1962) bzw. im Zellstoffwechsel (Garfinkel 1963) ergeben. Das Computer-Modell, gegeben durch Programm, Komplexität der Schaltung und Schaltelemente, eignet sich so zum Studium aller einschlägigen Fragen wie Absorption, Verteilung, Ausscheidung, Tole-

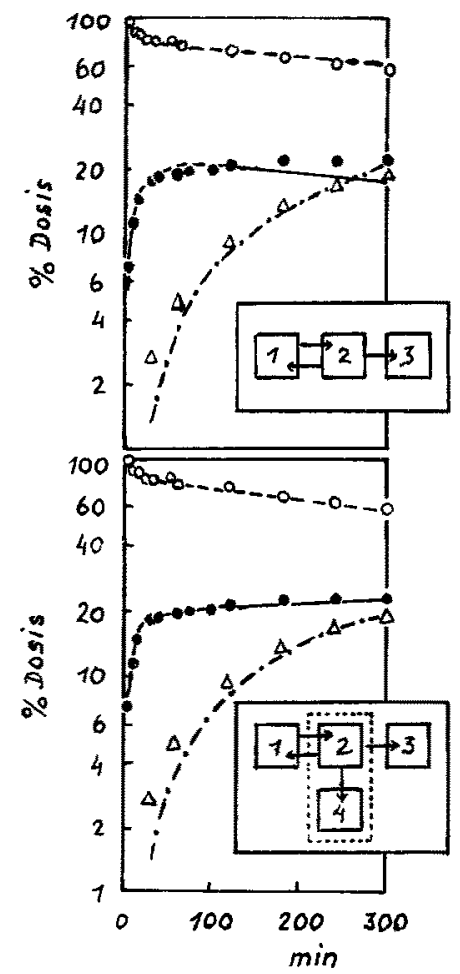

Abb. 3: Kompartimentalmodelle des Tyroxinstoffwechsels (nach HazeLRIG 1964, umgezeichnet und vereinfacht). Oben: 3-Kompartimentalmodell; unten: 4-Kompartimentalmodell. Meßwerte: 0 Thyroxin im Blut, $\bullet$ in der Leber, $\triangle$ als Summe von Galle und Jodid. Vom Modell vorausgesagte Werte (als Kurven):--- Thyroxin im Blut, _ in der Leber, -.-.-. als Summe yon Galle und Jodid

ranz, Zeitverzögerung, Induktion (adaptiver Enzymbildung) und ganz allgemein von Stoffwechsel, und bei bestimmten angenommenen Anfangsgrößen (Konzentration, Geschwindigkeitskonstanten, etc.) wird das Resultat als Kurve von Konzentration gegen Zeit geliefert. Da die Zeitfunktionen durch LAPLACE-Transformationen vereinfacht darzustellen sind, können durch Simulation unter bestimmten Annahmen auch die Beziehungen zwischen "input“, „output“ und Systemfunktion (Ubertragungsfunktion) studiert werden, so wenn beispielsweise durch eine schnelle intravenöse Injektion eine Impulsfunktion mit dem numerischen Wert 1 gegeben ist und die Plasmakonzentration als "output“ gleich der Systemfunktion wird. In diesen Modellen wird meist Kinetik 
1. Ordnung angenommen; die Einbeziehung komplizierterer kinetischer Annahmen führt zur Erhöhung der Komplexität.

An einem digitalcomputer-modellierten Kompartimentalsystem des Thyroxinstoffwechsels kann ein Beispiel für Simulation gegeben werden (HAzelRIg 1964): Bei einem zunächst gegebenen linearen 3-Kompartimentalsystem wird Verteilung von ${ }^{131} \mathrm{~J}$-Thyroxin im Blut (1. Kompartiment), steady-state-Austausch mit der Leber (2. Kompartiment) und Ausscheidung als Summe von Galle und Jodid (3. Kompartiment) angenommen. Ein Vergleich der experimentell direkt bestimmten Werte mit den durch das Computer-Modell vorhergesagten erfolgt unter Anwendung der Theorie der kleinsten Quadrate in der Weise, daß die Geschwindigkeitskonstanten solange durch ein systematisches Raten angepaßt werden, bis die Summe der Quadrate der Abweichungen zwischen theoretischen und experimentellen Punkten zu einem Minimum wird (Ackerman \& Hazelrig 1964). Die im 3-Kompartimentalmodell noch bestehende Inkonsistenz $\mathrm{z}$ wischen beiden kann durch ein 4-Kompartimentalmodell praktisch zum Verschwinden gebracht werden (Abb. 3), was bedeuten mag, daß vielleicht Thyroxin über die Leber mit der Schilddrüse ausgetauscht wird. Durch Änderung der Maschinenzeit des Computers im Vergleich zur aktuellen Zeit können auch anderswie nicht eruierbare Vorgänge, wie Oszillieren einiger Intermediate bei Enzymreaktionen, erkannt werden (WALTER 1966).

\section{KINETISCHE UND KYBERNETISCHE STOFFWECHSELMODELLE}

Wenn das Wesentliche eines Systems feststeht, kann es auf vielfältige Weise durch nicht nur mit ihm, sondern auch untereinander analoge Modelle realisiert werden. Auf der Basis von Differentialgleichungssystemen stellen kinetische und kybernetische Modelle, erstere durch kinetische Schemata, letztere durch Blockschaltbilder primär repräsentiert, solche einander analoge, wenn nicht sogar weitgehend äquivalente Modelle dar. Freilich darf streng genommen eine kybernetische Betrachtung des Stoffwechsels nicht einer kinetischen entgegengesetzt werden, da, wie schon erwähnt, die stofflichen Umsätze auf jeden Fall „informational“ gesteuert werden. Ein Zusammenhang zwischen Kinetik und Kybernetik ist bereits von DANZIGER \& ELMERGREEN (1957) gezeigt worden; er ergibt sich ganz eindeutig aus dem Flußdiagramm der (oszillierenden) Glykolyse nach HEss, Brand \& Pye (1966).

Immerhin mag es von Interesse sein, nach der Darstellung der typischen ReizReaktions-Gesetze (Locker 1964) durch ein regelungstheoretisches Modell zu fragen. Prinzipiell ließen sich diese aus dem kinetischen Ogston-LAIDLeR-Modell, das bei konstanter Enzymkonzentration unter dem Einfluß eines variierten Verhältnisses von Fremdstoff zu Substrat entweder einen mittleren Aktivitätszustand oder Hemmung beziehungsweise Aktivierung zeigt, ableiten. Diesem 4-gliedrigen kinetischen Modell kann ein kybernetisches (regelungstheoretisches) Modell zur Seite gestellt werden (Abb. 4), das in Anlehnung an Drischel (1952/53) gewonnen wurde, der es zur Veranschaulichung der antagonistischen Wirkung von Sympathicus und Parasympathicus (in der Funktionsweise des vegetativ-nervösen Zentralapparates) entwarf. Hierbei gelangt der vom Meßorgan stammende Signalstrom geteilt in 2 Halbzentren, die auf- 
einander einwirken, und von diesem zu einem gemeinsamen Stellglied. Von den ReizReaktions-Gesetzen kann das sogenannte WEBER-FECHNERsche Gesetz regelungstheoretisch möglicherweise als Kennlinie einer logarithmischen „input-output" mation ohne Sollwertverstellung interpretiert werden (unabhängig davon, daß heute mehr eine sogenannte Potenzfunktion im Sinne des Stevensschen Gesetzes im Vordergrund steht). Das WILDERsche Ausgangslagengesetz impliziert vielleicht einen Halteregler mit Störgrößenaufschaltung, der verschiedene Werte, von denen die Regelgüte des Systems abhängt, annehmen kann. Die Ausgangslage als Zustand größter Stabilität
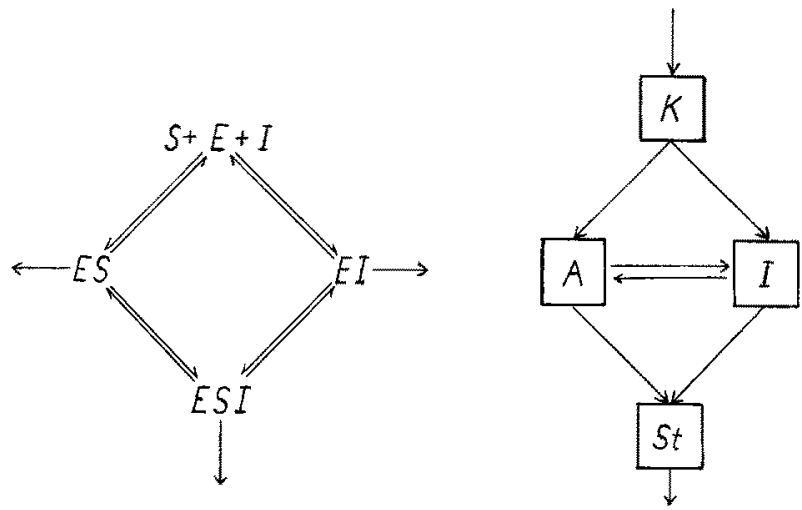

Abb. 4: Vergleich eines kinetischen Modells der Enzymaktivitär (das Hemmung und Aktivierung demonstrieren kann) mit einem regelungstheoretischen. Links: OGSTON-LAIDLER-Modell (nach Locker 1964, vereinfacht); rechts: im Anschluß an DrISCHEL (1952/53) skizziertes Modell. Hier wird der vom Meßorgan ausgehende Signalstrom durch den Kraftschalter (K) geteilt und in zwei Halbzentren von Stellmotoren weitergeführt (A: für Aktivierung, I: für Hemmung), die aufeinander einwirken können; von ihnen Ausgang zu einem gemeinsamen Stellglied (St)

läßt sich wohl versuchsweise auch mit dem Minimum der Regelfäche beim aperiodischen Grenzfall in Verbindung bringen. Schließlich kann vermutet werden, daß Verhalten nach dem ARNDT-Schulzschen Gesetz, also Aktivierung, eine Kennlinie voraussetzt, wie sie sich bei einem Halteregler mit vorübergehender Sollwertverstellung oder Störgrößenaufschaltung ergibt.

Ein sicher berechtigter Einwand gegen Heranziehung kybernetischer Modelle auf der Basis von Reglern für komplexe Stoffwechselvorgänge ist das Fehlen einer eindeutigen Zuordnung der Systembestandteile. Jedoch war die kybernetische Analogie solange verlockend und auch von Wert, als sie mangels besserer theoretischer Grundlagen die nach Beeinflussung stoffwechselnder Systeme auftretenden Schwingungsphänomene gut zu beschreiben vermochte, zum Beispiel als Übergangsfunktionen 2. Ordnung mit periodisch gedämpftem Einschwingvorgang oder mit reiner Differentialquotientenregelung. Jetzt, wo wir, wie noch zu erwähnen sein wird, über durchgearbeitete Modelle sogar für ungedämpfte Oszillationen verfügen, werden einfache regelungstheoretische Modelle entbehrlich; das gilt natürlich erst recht für die einfachen Analogien zwischen "over-shoot" oder "false start" und dem durch Addition 
oder Subtraktion des jeweiligen Anteils zu erklärenden Verhalten von PD-Gliedern. Trotzdem machen sowohl die oberflächlichen wie die feineren Analogien zwischen kinetischer und kybernetischer Betrachtung des Stoffwechsels einen epistemologisch bedeutsamen Sachverhalt sichtbar, nämlich daß die durch ein System gegebene Wirklichkeit letztlich nicht in den Griff zu bekommen ist.

\section{ZWISCHENBEMERKUNG ZU DETERMINISTISCHEN UND STOCHASTISCHEN MODELLEN}

Ein gewisser Ausweg bietet sich durch die Möglichkeit, gegebene Sachverhalte nicht als durchgehend deterministisch hinzunehmen, sondern zu ihrer Erkennung auch probabilistische (stochastische) Elemente in Rechnung zu stellen und daraus entsprechende Modelle zu bauen. Sie werden zwar die eindeutige Beziehung von „output“ $\mathrm{zu}$ „input “ vermissen lassen, dafür aber in anderer Weise nützlich sein können. Schon in regelungstheoretischen Modellen tritt dies, trotz ihrer weiten Anwendung namentlich in der Sinnesphysiologie, zutage, wenn es etwa bei einer dynamischen Kennlinienschar eine eindeutige Beziehung zwischen Reiz und Reaktion nicht gibt (RANKe 1960). Die Unerläßlichkeit stochastischer Modelle wird durch die Tracer-Kinetik dokumentiert, bei der es sich darum handelt, durch Zugabe einer radioaktiven Substanz nicht stationärer Konzentration etwas über die stationäre Population der Muttersubstanz auszusagen. Unter bestimmten einschränkenden Bedingungen (BERGNER 1964) kann aber der zeitliche Durchschnitt von Wahrscheinlichkeiten einer räumlichen Wahrscheinlichkeitsverteilung äquivalent sein, wie es durch ein Ergoden-Theorem (vgl. ARLEY \& Buch 1966) festgesetzt ist. So zeigt die Tracer-Kinetik, trotz der vorwiegend deterministischen Behandlung von Kompartimentalsystemen, stochastische Facetten.

Ein drittes Gebiet, in dem sich stochastische Modelle bereits Anerkennung erworben haben und einige von diesen deterministischen Ansätzen direkt äquivalent sind, ist das des biologischen Wachstums. Obwohl die stochastische Behandlung sich vorwiegend auf sogenannte äußere oder Makromodelle, nämlich Populationen von Individuen, bezieht, kann sie unschwer auf sogenannte innere oder Mikromodelle übertragen werden, weil ja Organismen Populationen von Zellen sind. Sie entgeht dem hauptsächlichen Einwand, der gegen ein deterministisches Modell erhoben werden kann, daß nämlich schwerlich die Organismusgröße zur Zeit $t$ bei gleichbleibenden Anfangsbedingungen immer dieselbe ist, durch Einbeziehung zufälliger Fluktuationen. Vielleicht kann die Wachstumstheorie, um deren Neufundierung sich in letzter Zeit KRüGER (1964) so verdient gemacht hat, durch Vergleich der deterministischen mit der stochastischen Betrachtungsweise, besonders im Hinblick auf die Wachstumsbedingungen zum Ausdruck bringende „output-input“-Beziehung profitieren. Gerade die für das Wachstum postulierte „Aquifinalität" wird bei größeren Fluktuationen in Frage gestellt werden müssen. 


\section{METABOLISCH BEDEUTSAME SYSTEMEIGENSCHAFTEN}

\section{Stabilität}

In linearen Reglern wird unter Stabilität eine beschränkte Lösung der entsprechenden Differentialgleichungssysteme verstanden; bei Übergang zur Instabilität werden unabhängig vom „input" selbsterregte Schwingungen ausgeführt. Im stoffwechselnden kinetisch betrachteten System wird mit Stabilität die Eigenschaft der Homöostase in Verbindung gebracht, der entsprechend aus einer großen Vielfalt von „inputs" ein einförmiger gewünschter "output" resultiert. Diese passive Stabilität wurde von MorowITz et al. (1964) durch Computer-Simulation eines einfachen biochemischen Modells studiert. Die Stabilität erweist sich als um so höher, je mehr Verknüpfungen zwischen den Elementen vorliegen und um so näher sich diese zum „output" befinden. In dieser Untersuchung war neben der Definition eines dimensionslosen Maßes für Stabilität auch der "Gestalt" des Systems Rechnung getragen worden. Der wichtigste die Stabilität beeinflussende Parameter ist die Verknüpfung (Konnektivität), das heißt die Anzahl und "Organisation" von graph-theoretisch nicht voll erfaßbaren Verbindungen zwischen den Systempunkten. Zwischen Konnektivität, Stabilität und Effektivität besteht folgende Beziehung: Ein "unverbundenes" System ist insofern maximal wirksam, als es mit einem Minimum an Enzymen auskommt; es wird aber zugleich maximal verwundbar. Ein stark oder maximal verbundenes System ist außerordentlich stabil; die Unterbrechung eines Abbauwegs in ihm erhöht den Durchsatz durch andere und die „over-all“-Geschwindigkeit bleibt dadurch unverändert. Allerdings muß hier eine große Anzahl von Enzymen vorhanden sein, mit deren Wirkung eine gewisse Verzögerung („lag-time") in Verbindung steht. Sonach erscheint Stabilität innig mit Komplexität und Organisation des Systems verbunden. Hohe Konnektivität ist in Systemen mit Folgereaktionen gegeben, weshalb diese stabil sind und sich automatisch auf einen stationären Zustand einstellen (Higgrns 1963). Es läßt sich zeigen, daß durch die Produktion von Stoffen im System Instabilitäten auftreten können (RASHEvSkY 1960).

\section{Übergänge, Feedback und Kontrolle}

Für die Bewegungszustände (Übergänge) in der Zelle können die charakteristischen Frequenzen angegeben werden, worunter die Kehrwerte der charakteristischen Zeiten zu verstehen sind, das heißt der Zeiten, innerhalb derer ein Übergang zwischen zwei Zuständen zu 50\% vollzogen ist (Hrggrns 1965). An Hand der charakteristischen Frequenzen läßt sich ein Niveau-Diagramm (Abb. 5) aufführen, in welchem alle Variablen unterhalb eines bestimmten Niveaus als konstant angesehen werden können: die sogenannten strukturellen Kontrollvariablen, die wie Geschwindigkeitskonstanten, Enzymkonzentrationen usw. die kinetischen Gleichungen bestimmen. Die Variablen oberhalb des untersuchten Niveaus sind sogenannte verborgene Variablen, sie werden nicht erregt, sind daher wirkungslos und befinden sich in einer dynamischen Substruktur. Trotz gewisser Einschränkungen ist praktisch jede Form von Übergang möglich; die Bewegung von einem durch die Anfangsbedingungen bestimmten Zustand zu einem 
neuen ist durch die Verminderung der Zahl von dessen Anfangsbedingungen gekennzeichnet. Die Kontrollvariablen sind im allgemeinen von vergleichbarer Größe; lediglich, wenn eine unter ihnen besonders groß ist, wird von dieser eine Flußkontrolle über andere Variable ausgeübt. Das bekannte „master-principle“ erfährt hier eine kinetische

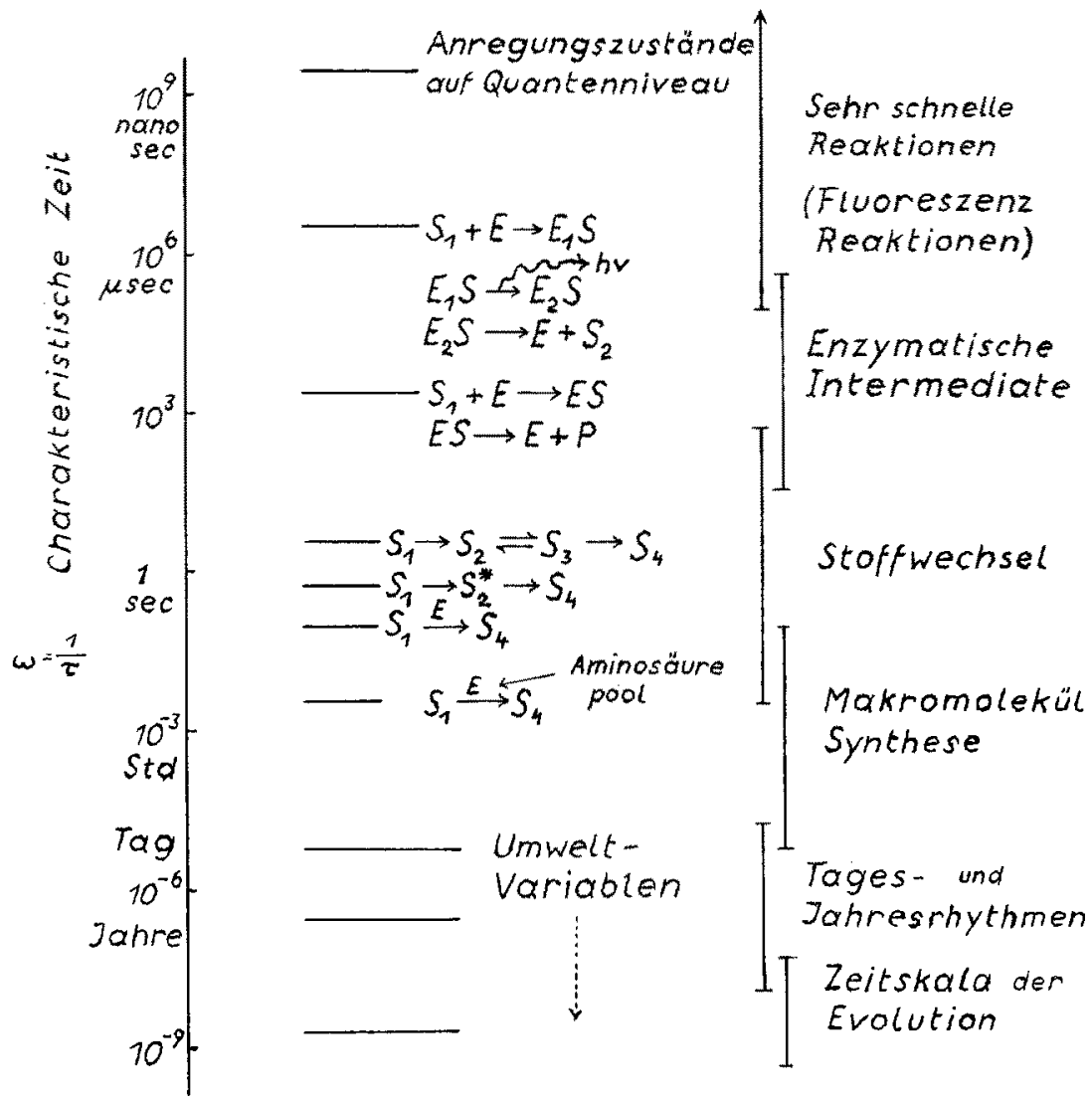

Abb. 5: Schichtendiagramm der die Zellbewegungen (Ubergänge, Rhythmen) beeinflussenden dynamischen Zustände (nach Higgins 1965, umgezeichnet und vereinfacht). In jeder Schicht, die oberste ausgenommen, nehmen die Bewegungsgleichungen gewöhnlich eine reduzierte Form von den für die darüberliegende Schicht gültigen Gleichungen an (Prinzipielle Rückführbarkeit des Massenwirkungsgesetzes auf die Quantenmechanik, der Stoffwechselgleichungen auf das Massenwirkungsgesetz etc.). Es ist fraglich, ob diese "Reduktion" für tiefere Schichten, wegen der mit ihnen verbundenen Organisation, gilt. $\omega$ : Charakteristische Frequenz, $\tau$ : Charakteristische Zeit (im Sinne der Relaxationszeit)

Verfeinerung. Rein regelungstheoretisch entsprechen diesem Prinzip des Schrittmachers die verschiedenen Formen von Kopplungen (bzw. relativer oder absoluter Koordination), nicht-lineare Wechselwirkungen von Steuergliedern aufeinander, die besonders, wie gleich zu zeigen ist, bei Oszillatoren wirksam werden.

Wenn zugleich mit der Flußkontrolle negativer Feedback auftritt, also beispielsweise eine Variable ihre eigene Nettoproduktion hemmt, weist das System wieder hohe 
Stabilität auf, wie es auch für ein lineares Feedback-System mit variierenden Parametern von $Z_{A D E H}(1951)$ gezeigt worden ist. Abhängig von der Lage und Struktur der Verbindungen zwischen den Variablen können nicht-oszillatorische oder oszillatorische Eigenschaften hervortreten.

\section{Oszillationen und ihre thermodynamischen Vorausetzungen}

Die Möglichkeit periodischer Stoffwechselreaktionen wurde schon von LOTKA (1910) erkannt; dieses Problem wurde unter anderem von Hearon (1953) weiterbearbeitet; heute liegt bereits eine umfangreiche Literatur vor und es ist sichergestellt, daß die für die Einstellung auf ein steady-state so wichtigen Ubergangsfunktionen mit (permanenten oder gedämptten) Oszillationen verlaufen. Allerdings sind für konservative und nicht-konservative Systeme, das heißt Systeme, deren Gesamtmasse und Energie in der Zeit varïert, die Verhältnisse nicht die gleichen. Für erstere finden sich, wie Goodwin (1965) zeigte, auch bei Nicht-Linearität im steady-state selbst keine Oszillationen; diese treten vielmehr erst nach minimalen Einflüssen im Sinne von „Rauschen“ auf und vermögen sich bis zur Instabilität zu verstärken. Auch diese an nicht-wechselwirkenden oder wechselwirkenden Feedback-Modellen gemachten Untersuchungen waren nur durch Computer möglich. Von Goodwin wurde auch eine der Temperaturfunktion analoge thermodymamische Größe, die sogenannte "talandische Temperatur als Maß der Schwingungsamplitude eingeführt, um bei der Unzahl von Kontrollschaltungen im Stoffwechsel eine dynamische Analyse wenigstens nach Art der statistischen Mechanik durchführbar zu machen. Bei Wechselwirkung zwischen Oszillatoren tritt neben Frequenzübertragung das Phänomen der subharmonischen Resonanz ein, wobei sich etwa bei ursprünglich schnellerer Frequenz die nunmehr verlangsamte Frequenz als Subharmonische überlagert. Durch Wechselwirkung von Oszillatoren kann eine Vielfalt von Frequenzen erzeugt werden, aus welcher sich durch besondere Kopplung die gewünschte auswählen läßt, wie auch Amplitudenerhöhung hervorgerufen werden kann und Aktivitäten genau miteinander in Phase kommen können. Das Auftreten einer subharmonischen Relation steht in Abhängigkeit von der Amplitude des freien Oszillators; bei kleiner Amplitude tritt keine Subharmonische auf. Die "talandische" Temperatur als Maß der Amplitude steht in direkter Beziehung zur Nicht-Linearität des Oszillators, weshalb ein Oszillator mit kleiner Amplitude fast linear ist und quasi-sinusförmig schwingt.

Im Vergleich zu konservativen Systemen mit permanenten Oszillationen ohne Dämpfung stellt Dämpfung, die in nicht-konservativen Systemen mit Grenz-ZyklusEigenschaften vorkommt - wobei der Grenz-Zyklus Lösungen gewisser nicht-linearer Differentialgleichungen bringt -, den allgemeineren Fall dar. In solchen Systemen können periodische Bewegungen bei allen Anfangsbedingungen nur über einem beschränkten Parameterbereich beobachtet werden. Die Ursache für das Auftreten von Schwingungen ist hier eine Zeitverzögerung in der Signalübermittlung - die. lange bekannte Tatsache, daß Schwingungen beispielsweise bei biochemischen Reaktionen nur bei mit Verzögerung wirkender Rückkopplung auftreten. 
Von Glansdorfa \& Prigogine (1954) wurden die Schwingungen im Zeitverhalten eines offenen irreversiblen Systems durch ein allgemeines Bewegungstheorem erklärt. Aber dieses Theorem ist nur zugleich mit der Annahme der phänomenologischen Gesetze, das heißt von linearer Beziehung $z$ wischen thermodynamischen Flüssen und Kräften und der Onsager-Relation gültig. Tatsächlich kann aber eine zyklische Bewegung, die vom Systempunkt im Phasenraum der thermodynamischen Kräfte ausgeführt wird, zum Unterschied von der Gültigkeit der phänomenologischen Gesetze entsprechend weit vom Gleichgewicht entfernt sein. Prigogine \& Balescu (1956) haben gezeigt, daß in der Nachbarschaft des stationären Zustandes ein stabiler zyklischer Prozeß ablaufen kann, der sich unendlich wiederholt, ohne in den stationären $\mathrm{Zu}$ stand selbst überzugehen. Die Voraussetzung für geschlossene Konturen des GrenzZyklus um den stationären Zustand sind antisymmetrische phänomenologische Koeffizienten und nicht symmetrische wie in der Onsager-Beziehung. Bernhard (1964) schlägt daher ein Grenz-Zyklus-Modell der periodischen Schwingungen vor, das nicht, wie es bisher geschieht, einen stabilen stationären Zustand annimmt, um den herum zyklische Bewegung stattfindet, sondern in welchem ein unstabiler Fokus von stabilen Grenzzyklen umgeben wird. Dieses Modell würde implizieren, daß die Zelle ohne zyklische Oszillationen überhaupt kein steady-state aufweisen kann.

\section{ENERGIE, INFORMATION UND STRUKTUR}

Die eben erwähnten thermodynamischen Voraussetzungen für Oszillation liefern neue Nahrung für schon of geäußerte Zweifel an der Gültigkeit des PrigogINeTheorems, wonach während des Übergangs eines offenen Systems auf ein steady-state (Fließgleichgewicht) seine Entropie abnehmen kann. Insbesondere ist bezweifelt worden (Foster, Rapoport \& Trucco 1957, Katchalsky \& Kedem 1962), daß bei Fehlen von Linearität und Symmetrie der Koeffizienten vorhergesagt werden kann, ob das System einen stationären Zustand erreicht und die Forderung minimaler Entropieproduktion erfullt. Neuerdings legt Trincher (1965) Nachdruck auf die Tatsache, daß das PRIgogine-Theorem deswegen nicht generell gelten kann, weil es während der Embryogenese zu einem spezifischen Anstieg der Entropieproduktion kommt - erst nach der ontogenetischen Herausbildung der Strukturen tritt das Gesetz der Stoffwechselreduktion in Kraft, demgemäß der Stoffwechsel mit der Körpergröße abnimmt (Locker 1961). Bei Trincher liegt die Attacke gegen das Prigogine-Theorem in einer Linie mit der Wiederaufnahme von seit langem angestellten Betrachtungen über die Zusammenhänge zwischen Energie und Information.

Bereits von SzILARD (1929) erkannt und von BRILLOUIN (1951) exakt festgelegt ist die Tatsache, daß aus Beobachtung über ein System gewonnene Information zur Abnahme von Entropie führt, daß aber durch die mit der Beobachtung verbundene Beeinflussung des Systems im Gesamtsystem (das aus ursprünglichem System und Beobachter besteht) die Entropie zunehmen muß. Dies ist, bei aller Problematik des PRIGOGINE-Theorems, doch eine gewisse Entsprechung zum thermodynamischen Verhalten eines offenen Systems, für welches allein die Entropie abnehmen kann, für welches zusammen mit seiner Umwelt sie immer zunehmen muß. Gleichwertigkeit von 
Neg-Entropie und Information wird von VALENTinuzzi (1962) zur Grundlage einer systematischen Studie genommen, wobei die bereits von LINSCHITz (1953) aufgestellten, aber nur für ideale Systeme gültigen Beziehungen eine auch auf reale physikalische Systeme anwendbare Generalisierung erfahren (vgl. auch BEIER 1965). Auf Grund dieser Untersuchungen kann nicht nur der strukturelle Informationsgehalt von Molekïlen, sondern möglicherweise auch von Systemen abgeschätzt werden.

Die Gegenüberstellung von „Energie“ und „Information“ findet sich im schon früher angestellten Versuch, kinetische und kybernetische Beschreibung des Stoffwechsels zu vergleichen, präformiert. Die Nebeneinanderstellung beider Betrachtungsweisen läßt die formalen Ahnlichkeiten in der „Operation“ der Systeme erkennen: der „inputoutput"-Beziehung allgemeinster Art entspricht im kinetischen System Import und Export von Material (und Energie), im kybernetischen System Eingang und Ausgabe von Information. Ein Regelkreis ist zunächst geschlossen und wird nur durch Aufschneiden offen; Offenheit für Nachricht (Information) ist nicht die gleiche wie für Material; durch "informationale" Einflüsse auf letzteres wird zwischen beiden Systemen eine Verbindung geschaffen. Beiden liegt somit etwas über Energie (Substanz) beziehungsweise Information Hinausgehendes zugrunde, nämlich "Struktur". Sie wird beispielsweise als die Art, wie Elemente zusammenhängen, definiert (WIENER 1958) und ist von der "inneren Natur" dieser Elemente unabhängig. Werden die Elemente Gegenstand einer Elementenstatistik, indem aus den relativen Häufigkeiten oder Wahrscheinlichkeiten, mit denen die Elemente einer Struktur auftreten, quantitative Ergebnisse gewonnen werden, gelangt man zunächst wieder zur Informationstheorie als einer allgemeinen Strukturtheorie; daher konnte Lutz (1963) zur Ordnung von Strukturen die SHannon-Formel verwenden. Will man von der Struktur zur Organisation als Ausdruck der Strukturkomplexität gelangen, muß man bedenken, daß die internen Korrelationen zwischen den Elementen zu Redundanz führen, weshalb die in einem System enthaltene Information nicht stets Anzeichen für Ordnung oder Organisation der Elemente sein muß (MARgalef 1958). Daher kann ein aus wenigen Elementen bestehendes System weniger wahrscheinlich als ein solches mit einer großen Elementenanzahl sein und enthält deswegen mehr Information, obwohl aus dem in usueller Weise errechneten Informationsgehalt gerade das Gegenteil hervorgehen kann. Weil die so errechnete Information in umgekehrtem Verhältnis zur Organisation des Systems stehen kann und eher Entropie als Information darstellt, spricht man hier nach Margalef (1958) besser von Mannigfaltigkeit. Ein System mit geringerer Mannigfaltigkeit besitzt eine Struktur mit mehr Ordnung (in sich) und mit geringerer Wahrscheinlickkeit; bereits eine kurze Reihe von Entscheidungen führt zur Kenntnis von ihm. Jedoch resultiert aus der Feststellung Brillourns (1951) die Tatsache, daß die Struktur eines materiellen Systems nur durch Wechselwirkung mit anderen Systemen gleicher Art erforscht und beschrieben werden kann - eine so durch Wechselwirkung definierte Struktur (ELSASSER 1958) führt bereits über den rein informationstheoretischen Aspekt hinaus. Die in das lebende System durch die einverleibten Moleküle hineinkommende Information ist gering im Vergleich zu der bereits in ihm enthaltenen; durch den Stoffwechsel wird das Informations-Potential konstant erzeugt und erhalten, worauf der Unterschied zwischen Organismus und Maschine beruht (TRINCHER 1965). Wohl sind beide thermodynamisch offene Systeme; während aber beim Organismus innere Arbeit (zur Wieder- 
herstellung der zerstörten Strukturen und zur Aufrechterhaltung des Informationsgehalts) und äußere Arbeit gleichzeitig ablaufen, sind sie bei der Maschine voneinander unabhängig und finden auch zu verschiedenen Zeiten statt - nur bei einer idealen Maschine kann erstere Quantität überhaupt vernachlässigt werden.

Beruht aber nicht bloß die Erforschung der Struktur auf Wechselwirkung des Systems mit dem Beobachter, sondern ist sie selbst und ihre Komplexität Ausdruck dynamischer Wechselwirkung zwischen den Elementen eines Systems, dann ist die rein informationstheoretische Behandlung unzureichend, da sie diese dynamischen Beziehungen ignoriert und beispielsweise durch Annahme bestimmter Wahrscheinlichkeiten für die Ereignisse (Morowrtz 1955) lediglich zu einer über den Dynamismus des Systems nichts aussagenden $\mathrm{Maßzahl}$, wie Informationsgehalt, kommt. In dynamischen Systemen ist Struktur von Information zu trennen. Dies wird ersichtlich, wenn man Information als die zur Koordination der Aktivität eines Systems notwendige Nachrichtenübertragung zwischen den Elementen ansieht. Bei gegebenen Aufgaben, wie Optimisierung des Systems, nimmt bei Vorliegen von nur ungenauer Information der Systembestandteile voneinander der Wirkungsgrad des Systems ab; die Zusammenhänge zwischen Struktur und Kommunikation im "operationalen“ System lassen sich direkt messen (Sengupta \& Ackoff 1965).

Gegenüber dem Begriff der "Struktur" in einem formalen System, wo er die Totalität der Relationen zwischen Subsystemen oder Elementen, die durch Dekomposition des Systems freigesetzt werden (TODA \& SHUFORD 1965), angibt, erfährt in einem realen oder idealen System der Begriff der "Information“ durch seine Verfeinerung als Informationsgehalt, Neg-Entropie und Neg-Information eine besondere, im Verhältnis zu „Energie“, dem Entropiegebiet, klar gekennzeichnete Stellung (BEIER 1965). Auch in dynamischen (kinetischen) Systemen, die nicht allein durch Logik oder Informationstheorie beschrieben werden können, wird die Struktur durch mathematische, also ideale Relationen, wie die der Netzwerktheorie oder letztlich der Mengenlehre als allgemeinster mathematischer Theorie dargestellt und ihr Verhalten durch Zeitfunktionen bestimmt. Der Sinn einer systemtheoretischen Betrachtung des Stoffwechsels darf sonach neben einem gewissen heuristischen Wert vielleicht vor allem in der theoretischen Bedeutung gesehen werden, die im Sichtbarmachen des hinter den Primärgegebenheiten befindlichen Allgemeinen, Formalen, Idealen auch für die Naturwissenschaft liegt.

\section{ABSCHLIESSENDE BEMERKUNGEN}

Es ist erfreulich, wie immer mehr, auch von Biochemikern, erkannt wird, daß im Begriff der "Information" sich zu den vertrauten Begriffen von Materie (Substanz) oder Energie etwas anderes, gewissermaßen "Geistiges" hinzugesellt (Schramm 1964). Auch die moderne erkenntnistheoretische Strukturforschung kommt zur Schlußfolgerung (Leinfellner 1965), daß in einem idealen Raum die Strukturen (Formen) „aller möglichen Welten " gegeben sind - dementsprechend untereinander analog beziehungsweise isomorph. Die Einordnung empirisch gewonnener Strukturen in mathematische also der Vorgang der Mathematisierung der Wissenschaft - entspricht somit dem idea- 
len Erkenntnisziel, dem Bestreben, die ideale Welt zu berühren. Wenn hierbei offenbar wird, daß scheinbar unabhängige Wege, wie Allgemeine Systemtheorie und Erkenntnistheorie zueinander konvergieren, dann ist das Postulat der LeIbNizschen „mathesis universalis" oder der KanTschen Metaphysik als Fundament jeder möglichen Naturwissenschaft wieder der Erfüllung nähergebracht. Für diesen Vorgang der Wiederfindung des Geistes ist charakteristisch, daß mit Hintikka (1966) von „KANT vindicated" gesprochen werden kann. Die Allgemeine Systemtheorie v. BerTaLANFFYs (1950a, b) hat, besonders da sie sich mit ihren vielfältigen Ansätzen an konkreten Problemen, wie hier denen des Stoffwechsels, bewährt, für diese Entwicklung als Meilenstein zu gelten.

\section{ZUSAMMENFASSUNG}

1. Bestehende Ansätze zur mathematischen Darstellung der Allgemeinen Systemtheorie auf mengentheoretischer und graph-theoretischer Grundlage werden zur Erörterung abstrakter Systeme genommen; ihre Anwendbarkeit auf Stoffwechselprobleme ist beschränkt.

2. Die mathematisch gut durchgearbeiteten Kompartimentalsysteme eignen sich demgegenüber zur Analyse der Tracer-Kinetik und von zellulären Stoffwechselvorgängen.

3. Die Bedeutung konkreter physikalischer Computer-Modelle zum Studium (der Simulation) von Stoffwechselproblemen wird an einigen Beispielen eingehend besprochen.

4. Stoffwechselmodelle auf kinetischer und kybernetischer Basis werden miteinander verglichen, die Analogie von deterministischen und stochastischen Modellen herausgestellt und metabolisch bedeutsame Systemeigenschaften wie Stabilität, Kontrolle und Oszillationen gesondert beschrieben.

5. In die Diskussion der den Modellvorstellungen inhärenten Begriffe von "Information", "Energie" und "Struktur" werden einige epistemologische Fragen eingeschlossen.

\section{ZITIERTE LITERATUR}

Ackerman, E. \& Hazelrig, J. B., 1964. Computer applications to the evaluation of dynamic biological processes. In: Dynamic clinical studies with radioisotopes. Ed. by R. M. Kniseley \& W. N. Tauxe. U.S. Atomic Energy Commission, Washington, D.C., 19-53.

ARLEY, N. \& BUCF, K. R., 1966. Introduction to the theory of probability and statistics. Wiley $\&$ Sons, New York, 240 pp.

Ashbx, W. R., 1961. General system theory and the problem of the black box. In: Regelungsvorgänge in lebenden Wesen. Hrsg. v. H. Mittelstaedt. Oldenbourg, München, 51-62.

BEIER, W., 1965. Einfuhhrung in die theoretische Biophysik. G. Fischer, Stuttgart, 237 pp.

Bergner, P. E., 1964. Kinetic theory. Some aspects of the study of metabolic processes. In: Dynamic clinical studies with radioisotopes. Ed. by R. M. Kniseley \& W. N. Tauxe. U.S. Atomic Energy Commission, Washington, D.C., 1-18.

Berman, M., 1963. The formulation and testing of models. Ann. N.Y. Acad. Sci. 108, 182-194. 
BERNHARD, R., 1964. Survey of some biological aspects of irreversible thermodynamics. J. theoret. Biol. 7, 532-557.

BerTalanffy, L. von, 1950a. An outline of general system theory. Br. J. Phil. Sci. 1, 134-165.

- 1950b. The theory of open systems in physics and biology. Science, N.Y. 111, 23-29.

Briluouin, L., 1951. Maxwell's demon cannot operate. J. appl. Pbys. 22, 334-343.

Chance, B., Higgins, J. J. \& Garfinkel, D., 1962. Analogue and digital computer representations of biochemical processes, Fed. Proc. Fedn Am. Socs exp. Biol. 21, 75-86.

Danziger, L. \& Elmergreen, G. L., 1957. Mathematical models of endocrine systems. Bull. math. Biophys. 19, 9-18.

Drrschel, H., 1952/53. Bausteine einer dynamischen Theorie der vegetativen Regulation. Wiss. Z. Univ. Greifswald 2, 99-163.

Eilenderg, S. \& Maclane, S., 1945. General theory of natural equivalences. Trans. Am. math. Soc. 58, 231-294.

Elsasser, W. M., 1958. The physical foundation of biology. Pergamon pr, London, $219 \mathrm{pp}$.

Foster, C., Rapoport, A. \& Trucco, E., 1957. Some unsolved problems in the theory of non-isolated systems. Gen. Syst. 2, 9-29.

Garfinkel, D., 1963. Digital computer simulation of systems apparently compartmented at the cellular level. Ann. N.Y. Acad. Sci. 108, 293-304.

Glansdorff, P. \& Prigogine, I., 1954. Sur les propriétés différentielles de la production d'entropie. Physica, 's Grav. 20, 773-780.

Goodwin, B. C., 1965. Oscillatory behavior in enzymatic control processes. Adv. Enzyme Regul, 3, 425-438.

Halr, A. D. \& FA.GEN, R. E., 1956. Definition of System. Gen. Syst. 1, 18-28.

HazelRig, J. B., 1964. The impact of high-speed automated computation on mathematical models. Proc. Staff Meet. Mayo Clin. 39, 841-848.

Hearon, J. Z, 1953. The kinetics of linear systems with special reference to periodic reactions. Bull. math. Biopbys. 15, 121-141.

- 1963. Theorems on linear systems. Ann. N.Y. Acad. Sci. 108, 36-68.

HEss, B., Brand, K. \& PYE, K., 1966. Continuous oscillations in a cell-free extract of S. carlsbergensis. Biochem. biopbys. Res. Commun. 23, 102-108.

Hugcrvs, J. J., 1963. Analysis of sequential reactions. Ann. N.Y. Acad. Sci. 108, 305-321.

- 1965. Dynamics and control in cellular reactions. In: Control of energy metabolism. Ed. by B. Chance, R. W. Estabrook \& J. R. Williamson. Acad, pr., New York, 13-46.

HintrkKa, J., 1966. Kant vindicated. In: Deskription, Analytizität und Existenz. Hrsg. v. P. Weingartner. Pustet, München, 235-253.

KäMMERER, W., 1966. Zum mathematischen Modell automatischer Systeme. Stud. Biophys. (im Druck)

Katchalsky, A. \& Kedem, O., 1962. Thermodynamics of flow processes in biological systems. Biopbys. J. 2, 53-78.

KR ÜGER, F., 1964. Neuere mathematische Formulierungen der biologischen Temperaturfunktion und des Wachstums. Helgoländer wiss. Meeresunters. 9, 108-124.

Leinfellner, W., 1965. Struktur und Aufbau wissenschafticher Theorien. Physica Verl., Wien, Würzburg, $307 \mathrm{pp}$.

Linschitz, H., 1953. Information and physical entropy. In: Information theory in biology. Ed. by H. Quastler. Urbana, Ill., Univ. pr., 14-15.

Locker, A., 1961. Das Problem der Abhängigkeit des Stoffwechsels von der Körpergröße. Naturwissenschaften 48, 445-449.

- 1964. Reaktionen metabolisierender Systeme auf experimentelle Beeinflussung, Reiz und Schädigung. Helgoländer wiss. Meeresunters. 9, 38-107.

- 1966. Die Bedeutung der Begriffe Ganzheit, System und Modell für die quantitative Biologie. Intern. Z. Vitalst. (im Druck)

Lotka, A. J., 1910. Contribution to the theory of periodic reactions. J. phys. Chem. Ithaca 14, $271-275$.

LuTz, TH., 1963. Einige Gesichtspunkte zum Begriff der Struktur. Grundlagen-Stud. Kybernetik Geisteswiss. 4, 1-6.

Margalef, D. R., 1958. Information theory in ecology. Gen. System 3, 36-71. 
Mesarovic, M. D., 1964. Foundations for a general systems theory. In: Views on general systems theory. Ed, by M. D. Mesarovic. Wiley \& Sons, New York, 1-24.

Morowitz, H. J., 1955. Some disorder-order considerations in living systems. Bull. math. Biophys. 17, 81-87.

- Higinbotham, W. A., Mathysse, S. W. \& Quastler, H., 1964. Passive stability in a metabolic network. J. theor. Biol. 7, 98-111.

Prigogine, I. \& Batescu, R., 1956. Phénomènes cycliques dans la thermodynamique des processus irréversibles. Bull. Acad. r. Belg. 42, 256-265.

Ranke, O. F., 1960. Physiologie des Zentralnervensystems vom Standpunkt der Regelungslehre. Urban \& Schwarzenberg, München, $133 \mathrm{pp}$.

Rashevsky, N., 1954. Topology and life. Bull. math. Biopbys. 16, 317-348.

- 1960. Mathematical biophysics. Dover publ., New York, vol. 1, 1-488.

- 1965. The representation of organisms in terms of predicates. Bull. math. Biopbys. 27, $477-491$.

Rescigno, A. \& Segre, G., 1961. The precursor-product-relationship. J. theoret. Biol. 1, $498-513$.

- - 1962. Analysis of multicompartmented biological systems. J. theoret. Biol. 3, 149-163.

Rosen, R., 1958a. A relational theory of biological systems. Bull. math. Biophys. 20, 245-260.

- 1958b. The representation of biological systems from the standpoint of the theory of categories. Bull. math. Biophys. 20, 317-341.

Schoenfeld, R. L., 1963. Linear network theory and tracer analysis. Ann. N. Y. Acad. Sci. 108, 69-91.

Schramm, G., 1964. Der Informationsgehalt von Nukleinsäuren. Dt. med. Wschr. 89, 65-72.

SengUPt A, S. S. \& Ackoff, R. L., 1965. Systems theory from an operations research point of view. Gen. Syst. 10, 43-48.

Sheppard, C. W. \& Housezolder, A. S., 1951. The mathematical basis of the interpretation of tracer experiments in closed steady-state systems. J. appl. Phys. 22, 510-520.

SugitA, M., 1961. Functional analysis of chemical systems in vivo using a logical circuit equivalent. I. theoret. Biol. 1, 415-430.

SzILARD, L., 1929. Uber die Entropieverminderung in einem thermodynamischen System bei Eingriff intelligenter Wesen. Z. Pbys. 53, 840-856.

TAYLOR, J. D. \& WIEGAND, R. G., 1962. The analog computer and plasma drug kinetics. Clin. Pharmac. Ther. 3, $464,472$.

TODA, M. \& SHuford, E. H., 1965. Logic of systems: Introduction to a formal theory of structure. Gen. Syst. 10, 3-27.

TRINCHER, K. S., 1965. Biology and information. Elements of biological thermodynamics. Consultants Bureau, New York, 93 pp.

Valentinuzzi, M. \& VAlentinuzzI, M. E., 1962. Information content of chemical structures and some possible biological applications. Bull. math. Biophys. 24, 11-27.

WALTER, Ch., 1966. Oscillation in enzyme reactions. Nature, Lond. 209, 404-405.

Wiener, N., 1958. Mensch und Menschmaschine (The human use of human beings). A. d. Am. Ullstein, Frankfurt a. M., 187 pp.

ZADEH, L. A., 1951. On stability of linear varying parameter systems. J. appl. Phys. 22, $402-405$.

ZEMANEK, H., KRETZ, H. \& ANGYAN, A. J., 1961. A model for neurophysiological functions. In: Information theory. Ed. by C. Cherry. Butterworth 8 Co., London, 270-284.

\section{Diskussion im Anschluß an den Vortrag Locker}

SCHARF: Es ist nicht erforderlich, eine Speicherung von Thyroxin in der Glandula thyreoidea anzunehmen. Nachweislich wird Thyroxin im Hypophysenvorderlappen-System aus dem Kreislauf herausgenommen, gespeichert und von dort in Umlauf gebracht.

LOCKER: Ich danke für diesen interessanten Hinweis.

Hess: Ich möchte zu dem sehr schönen Vortrag, der mich als Biochemiker interessiert hat, be- 
merken: Die Reaktionsweise der Enzyme ist mathematisch bekannt; man erhält aber mathematisch nur sinnvolle Lösungen, wenn man Kompartinente einführt. Unser ComputerModell der Atmung und Glykose enthält 3 Kompartimente für ATP und 2 Kompartimente für DPNH/DPN. - Ich habe den Eindruck, daß der Begriff des steady-state nicht sehr eindeutig definiert ist. Ein Vorgang, der stationär oszilliert, ist durchaus stationär.

LOCKer: Es ist durchaus richtig, daß die Notwendigkeit, Kompartimente anzunehmen, aus anomalen Situationen resultiert, aus Abweichungen zwischen experimentell bestimmten und computer-simulierten Werten, die nicht zu rechtfertigen sind und eben erst dann befriedigend klein werden, wenn man Kompartimente einführt. - Zugegebenermaßen ist es schwer, „steadystate $^{\text {"im }}$ im Stoffwechsel zu definieren. Schon rein kinetisch wird von manchen Autoren, so von HrggrNs (1963) eine feine, aber doch merkbare Unterscheidung zwischen steady-state und stationärem Zustand vorgenommen. Was die thermodynamische Bedingung des stationären Zustands anlangt, bin ich davon überzeugt, daß der Vorschlag Bernhards (1964), der bisherige Vorstellungen umkehrt und im stationären Zustand per definitionem Oszillationen auftreten läßt, nicht sdhnell allgemeine Anerkennung finden wird. Jedenfalls wird diese Frage noch weiterer theoretischer Bearbeitung bedürfen.

KRÜGER: Sie haben mich persönlich angesprochen. Im Gegensatz zur molekularen Basis habe ich versucht, biologische Phänomene im Makrobereich mathematisch zu beschreiben. Sie beziehen sidh auf das Wachstum und den Temperatureinfluß. Die von mir vorgeschlagene Wachstumsfunktion ist geeignet, das tierische Wachstum über sehr lange Zeiträume mit einer einfachen Funktion in sehr guter Näherung wiederzugeben. Mir kommt es darauf an, nachzuweisen, daß das Dogma der Biologen, daß biologische Phänomene einer mathematischen Verarbeitung nicht zugängig seien, falsch ist. Das Kriterium für eine mathematische Darstellung ist nicht nur die zahlenmäßige Wiedergabe eines Vorganges. Sie muß auch allgemeiner gültig sein, wie es zum Beispiel bei der allometrischen Funktion der Fall ist. Zweitens muß sie auch mathematisch definierte Querverbindungen gestatten, wie ich sie in der Deutung der Parameter der allometrischen Beziehung fand. Mit meiner Temperaturfunktion konnte ich mathematische Beziehungen zum Wachstumsvorgang aufweisen, auch wenn sie noch nicht vollkommen übersichtlich sind. Aud für die molekularen Ansätze wird eines Tages der mathematische Anschluß an die Phänomene im Makrobereich als Kriterium dienen.

LOCKER: Ihr Anliegen ist mir durchaus geläufig, und ich teile mit Ihnen die Ansicht, daß Bearbeitungen eines Problems vom Makrobereich aus sowohl als auch vom Mikrobereich irgendwo in der Mitte sich treffen müssen; allerdings wird man gerade für komplexe Phänomene und wer würde an der Komplexität des Wachstumsvorganges zweifeln - nicht zu einfache mathematische Modelle voraussetzen dürfen, selbst wenn wir in gewissem Sinne NEWTONS Ausspruch: "Natura enim simplex est" unterstreichen wollten.

SUGrTA: How to define thermodynamical functions, like chemical potential, is a very important problem of theoretical physics. The pessimistic opinions of biologists concerning mathematical methods will be reduced, if the exact definition were given. In the case of growth, the theory of small systems may be necessary, because molecular fluctuations may be large.

LOCKER: Das ist ja auch der Grund, warum ich für die Einführung einer stochastischen Betrachtung in die Theorie des organismischen Wachstums eintrete. 